hep-th/0406198

UFIFT-HEP-04-10

\title{
Cosmological Rescaling through Warped Space
}

\author{
Xingang Chen \\ Institute for Fundamental Theory \\ Department of Physics, University of Florida, Gainesville, FL 32611
}

\begin{abstract}
We discuss a scenario where at least part of the homogeneity on a brane world can be directly related to the hierarchy problem through warped space. We study the dynamics of an anti-D3-brane moving toward the infrared cutoff of a warped background. After a region described by the DBI action, the self-energy of the anti-D3-brane becomes comparable to the strength of the background. Then the world-volume scale of the anti-D3-brane is no longer comoving with the background geometry. After it settles down in the infrared end, the world-volume inhomogeneity will appear, to a Poincare observer, to be stretched by an exponentially large ratio. This ratio is close to that of the hierarchy problem between the gravitational and electroweak scales.
\end{abstract}




\section{INTRODUCTION}

Various hierarchies between different physical scales are among the most important challenges to any fundamental theory. Two of them related to this paper are in the context of the particles physics and cosmology. The former is the hierarchy problem between the gravitational and electroweak scale, which is about 32 e-folds. One natural interpretation to this problem was presented by Randall and Sundrum using a warped space [1]. Such a space can be realized in string theory [2-4] and has also played important roles recently in the studies of the de Sitter space construction $[5,6]$ and brane inflationary scenario [7-10].

The latter refers to the hierarchy between the homogeneous scale of our observable universe and the scale of the big bang cosmological causal contact. It is around 30 to 60 efolds depending on detailed cosmological evolution. This is usually addressed by inflationary scenario $[11] .{ }^{1}$ In this paper, we present a scenario where part of this hierarchy may be directly related to the one in the particle physics.

We make use of the abovementioned warped space. The string theory setups in those studies typically involve some anti-D3-branes located at the infrared (IR) cutoff of this warped background. The ultraviolet (UV) cutoff is smoothly glued into a compact manifold. We are living on the antibranes. Originally these antibranes should enter from the UV entrance and be attracted toward the IR end under the background gravitation and fiveform field. Starting with zero initial velocity, the longitudinal scale of the antibranes will first comove with the shrinking background and the inhomogeneities on the antibrane worldvolume keep the same wave-numbers to a Poincare observer. The proper energy density of the comoving antibranes will grow rapidly and cause a back-reaction on the background [15]. When the self-gravitational field dominates over the background field, the antibranes enter a non-comoving phase. Because of this phase, after the antibranes lose their kinetic energy and become at rest at the IR end, the antibrane world-volume embedding in the ambient space has been rescaled. From the point of view of the Poincare observer, the inhomogeneities on the world-volume have been stretched by an exponentially big factor, which is directly determined by the background geometry. This is close to the hierarchy ratio between the gravitational and electroweak scales. This mechanism, however, does not apply to the gravitational inhomogeneity in the background geometry.

\footnotetext{
${ }^{1}$ Other attempts include [12-14].
} 


\section{COMOVING BRANE SCALE IN THE WARPED SPACE}

We shall consider the dynamics of a single anti-D3-brane in most part of the paper. Having a stack of them for our purpose simply changes the antibrane tension. For simplicity we will neglect the gravitational coupling and consider only the flat 4-d space-time to illustrate the idea. This idea can be generalized to the gravitationally coupled case as well. An anti-D3-brane in a warped space

$$
d s^{2}=G_{M N} d X^{M} d X^{N}=h^{2}(r)\left(-d t^{2}+d \mathbf{x}^{2}\right)+h^{-2}(r) d r^{2}, \quad h(r)=r / R,
$$

with a four-form potential $C_{4}$ is described by the Dirac-Born-Infeld (DBI) plus Chern-Simons action $[16]$

$$
S=T_{3} \int d^{4} \xi\left[-\sqrt{-\operatorname{det}\left(\partial_{a} X^{M} \partial_{b} X^{N} G_{M N}\right)}-\frac{1}{4 !} \epsilon^{a_{1} \cdots a_{4}} \partial_{a_{1}} X^{M_{1}} \cdots \partial_{a_{4}} X^{M_{4}} C_{M_{1} \cdots M_{4}}\right]
$$

where $\xi^{a}(a=0,1,2,3)$ are the world-volume coordinates on the antibrane, and $X^{M}(M=$ $0,1,2,3,4)$ are the coordinates of the ambient $\mathrm{AdS}_{5}$ space. The first four components of $X^{M}$ are identified with $x^{\mu}(\mu=0,1,2,3)$ and the last $\left(X^{4}\right)$ with $r$ in (2.1). $T_{3}$ is the antibrane tension and $R$ is the characteristic length scale of the AdS space. Here the non-vanishing four-form potential components are $C_{0123}=h^{4}$. The $\xi^{a}$ dependence of $X^{M}\left(\xi^{a}\right)$ describes how the antibrane is embedded in the ambient space. We consider here a homogeneous probe anti-D3-brane along the $x^{\mu}$ directions. The $r$ is then the transverse position of this probe antibrane. We take the ansatz $X^{i}=s(t) \xi^{i}(i=1,2,3)$ and $X^{4}=r(t)$ and use the gauge $X^{0} \equiv t=\xi^{0}$.

The zero and fourth components of the equations of motion corresponding to the action $(2.2)$ are

$$
\begin{aligned}
s^{3} \frac{d}{d t}\left(\frac{h^{6}}{\sqrt{h^{4}-\dot{r}^{2}}}\right)+\frac{d}{d t}\left(s^{3} h^{4}\right) & =0, \\
\frac{d}{d t}\left(\frac{h^{2} \dot{r}}{\sqrt{h^{4}-\dot{r}^{2}}}\right)+\frac{2 h \partial_{r} h}{\sqrt{h^{4}-\dot{r}^{2}}}\left(2 h^{4}-\dot{r}^{2}\right)+\partial_{r}\left(h^{4}\right) & =0 .
\end{aligned}
$$

The other components vanish identically. We note that Eq. (2.4) is independent of the antibrane scale factor $s(t)$ and it gives rise to a conserved quantity

$$
\mathcal{E}=\frac{h^{6}}{\sqrt{h^{4}-\dot{r}^{2}}}+h^{4} .
$$

Differentiating both sides of Eq. (2.5) and using Eq. (2.3), we can see that $s(t)=$ const. For convenience we will choose $s(t)=1$ in the rest of the paper. It is then easy to check that (2.5) is in fact the coordinate energy density in unit of $T_{3}$, namely $\mathcal{E}=E / T_{3} V_{3}$. 


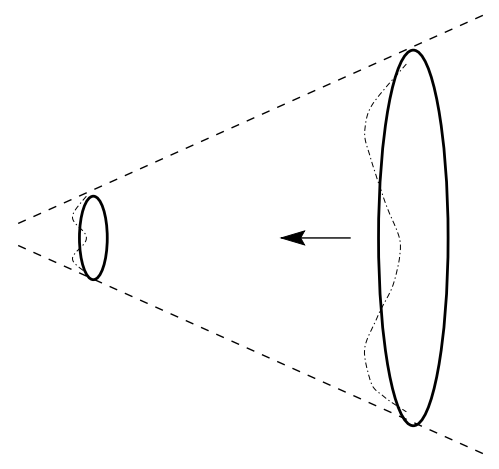

(A)

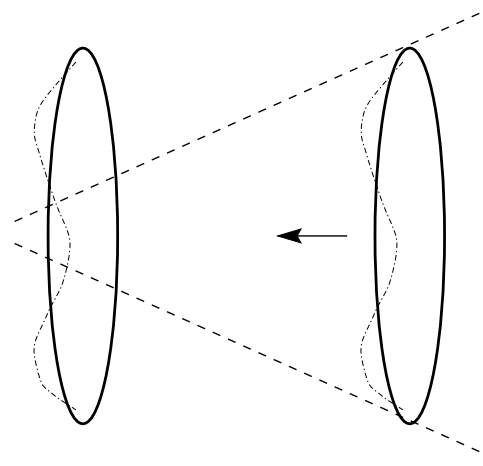

(B)

FIG. 1. The dashed lines are the background geometry. For illustration, we compactify the three spatial dimensions of the antibrane and show only one of them on the graph. The wiggles indicate the inhomogeneities. In (A), the world-volume scale comoves with the background geometry, while in (B), the background geometry is negligible to the antibrane.

In this paper we will be interested in the Poincare observer. This observer stays on the anti-D3-brane and uses the space-time coordinates $(t, \mathbf{x})$ defined in (2.1). To this observer, as long as the anti-brane is away from the UV entrance, the effective four-dimensional Plank mass is approximately constant as in the Randall-Sundrum model, while the string scale is red-shifting. ${ }^{2}$

The constant scale factor $s(t)$ means that, according to the DBI action, the scale of the antibrane is comoving with the background geometry. (See Fig. 1(A).) So if we consider a world-volume field perturbation, its wave-number remains the same to a Poincare observer. This conclusion holds for arbitrary $h(r)$ and $C_{0123}(r)$, and therefore also applies to D3-branes, as well as other more general background. This can also be straightforwardly generalized to the time-dependent background where one replaces the static flat metric $\eta_{\mu \nu}$ in (2.1) by $g_{\mu \nu}=\operatorname{diag}(-1, a(t), a(t), a(t))$.

The validity of the DBI action requires that the energy involved in the corresponding low-energy effective field theory be much smaller than the mass $\left(\sim r / \alpha^{\prime}\right)$ of massive Wbosons that have been integrated out, namely $\dot{r} / r \ll r / \alpha^{\prime}[16]$. It also requires that the back-reaction of the probe (anti)branes be negligible to the AdS background [15]. This condition will be important to our discussion.

\footnotetext{
${ }^{2}$ As we will discuss in Sec. IV\&V, when the back-reaction of the anti-D3-brane becomes strong and the DBI action fails, this definition should be understood as replacing the warped factor $h$ in (2.1) with an effective warped factor felt by the anti-D3-brane.
} 


\section{COMOVING REGION AND BACK-REACTION}

Consider an anti-D3-brane entering the warped space from the UV entrance $(r=R)$ with zero initial velocity $(\mathcal{E}=2)$. As the antibrane travels through the comoving region described by the DBI action in the last section, it experiences two different phases before the back-reaction becomes large. The first one is the non-relativistic comoving phase. This requires $\dot{r}^{2} \ll h^{4}$ and we can approximate Eq. (2.5) as

$$
\mathcal{E} \approx \frac{1}{2} \dot{r}^{2}+2 h^{4}
$$

So in this region, $r^{4} / R^{4} \approx 1$ and both constraints mentioned in Sec. II can be easily satisfied.

As $h^{4}$ decreases, the second term in Eq. (2.5) becomes negligible when $r^{4} / R^{4} \ll 1$. The antibrane are then entering the relativistic comoving phase. This region is also called the speed limit region and has been discussed in detail in $[17,15]$. The leading order of the antibrane motion is completely determined by the kinetic term in Eq. (2.5) and this asymptotic behavior is

$$
r \rightarrow \frac{R^{2}}{t}-\frac{R^{10}}{14 \mathcal{E} t^{9}}+\cdots
$$

Using this behavior, the first condition for the validity of the DBI action mentioned in Sec. II becomes $R^{2} \gg \alpha^{\prime}$, which can be easily met. However the second requirement, namely the smallness of the back-reaction of this probe antibrane, puts a strong constraint on the valid region of the DBI action [15].

To a Poincare observer, the antibrane tension $h^{4} T_{3}$ is red-shifting as it travels toward smaller $r$ region, while from (2.5) the total energy density $2 T_{3}$ is conserved. So at small $h^{4} \ll 1$, this antibrane is highly relativistic. In this process, the proper spatial volume of the antibrane shrinks and the energy becomes more concentrated. If we think of the AdS background as the near-horizon geometry of $N$ D3-branes ( $N$ is related to $R$ by $R^{4} \approx g_{s} N \alpha^{\prime 2}$ ) [2], and roughly treat the gravitational effect of this relativistic antibrane to be similar to $2 h^{-4}$ number of static antibranes, then in order to neglect the back-reaction we need $h^{4} \gg 2 / N$.

For example if $N \approx 10^{4}$, the relativistic comoving phase is within $10^{-1} \gtrsim r^{4} / R^{4} \gtrsim 10^{-3}$. For $r^{4} / R^{4} \lesssim 10^{-4}$, the DBI action starts to break down due to the antibrane back-reaction. The corresponding warping is approximately three e-folds. Since the order of the magnitude does not change much as long as $N$ is not extremely large, we will use this example in the following.

For later discussion, we comment that similar behavior applies to a D3-brane [15]. The difference is that the D3-brane carries the opposite RR charge so the Chern-Simons potential 
terms in Sec. II (such as the second term in Eq. (2.5)) change sign. Therefore D3-brane does not experience a net force in this background. An initial velocity is necessary for it to fall toward the IR end. For a small initial velocity $v \ll 1$, the relativistic comoving phase starts from $h^{4} \ll v^{2}$ and the back-reaction becomes important for $h^{4} \lesssim v^{2} / N$.

\section{NON-COMOVING ANTIBRANE}

In this section we explore a physical consequence after the antibrane back-reaction becomes large. We use the AdS background with an IR cut-off at $r=r_{I R}[3,4]$.

If we naively extend the comoving results of Sec. II until the warp factor $h$ is several e-folds below $N^{-1 / 4}$, the discussion in the last section shows that the scale of the energy density of the gravitational field of the probe antibrane is much bigger than that of the background. Therefore the self-gravitational field of the mobile antibrane dominates over the background and the background fields can be neglected. Effectively, the DBI action should be modified so that the induced metric becomes a spatial independent constant, whose value is roughly determined by the warp factor at the DBI break-down point. (See Fig. 1(B).) Realistically there should exist a region where the antibrane transits smoothly from the comoving region to this kind of behavior. For example in the next section, we will approximate it by a smooth effective geometry shown in Fig. 2.

Before this relativistic antibrane settles down in the IR end, energy is lost through the interactions with the background. The proper spatial volume $\left(\int h_{e}^{3} d^{3} \mathbf{x}\right)$ of the antibrane should remain approximately the same when it is losing its kinetic energy in the non-comoving region where the antibrane self-energy dominates. At the mean while, the decreasing antibrane self-energy causes the restoration of the background geometry. Eventually after the antibrane becomes at rest at the IR tip, we can again treat it as a probe antibrane. But the important thing is that the embedding of the antibrane in the ambient space has now been rescaled. To a Poincare observer, the initial inhomogeneities and homogeneous patches on the antibrane are stretched by an exponentially large rescaling factor. (The scalar field amplitudes are kept the same in the non-comoving region since in our normalization (2.1) they are proportional to $\Delta r$.)

According to Randall and Sundrum, to the Poincare observer, the shrinking of the effective background warp factor reduces the energy scale of the electroweak interaction relative to the gravitational scale. If at the UV entrance we assume that both of the scales are of the same magnitude, the final hierarchy is determined by the warp factor of the IR tip. In our scenario, part of the homogeneity and flatness of the universe is achieved in the same cosmological evolution. The rescaling factor is also completely determined by the background 
and is a few e-folds below the hierarchy ratio, since the non-comoving phase starts several e-folds away from the UV entrance.

We end this section with a few comments.

To have an efficient rescaling of the antibrane world-volume embedding, it is important that we assume most of the kinetic energy is lost in the non-comoving region. This is plausible since in this region the background is distorted by the antibrane and the interaction between them is maximum. But the details remain to be understood more explicitly.

This mechanism does not apply to any inhomogeneity existing in the AdS background, which may give rise to a spatial varying 4-d Planck constant. To a Poincare observer, this inhomogeneity does not rescale as the antibrane embedding scale changes.

\section{A SIMPLE MODEL}

Comparing to the DBI action, the detailed evolution of the process described in the last section is under less mathematical control so far. Here we give a simple model to describe a possible intermediate process. We note that, while some robust features of our scenario have been addressed in the last few sections, the more detailed evolution of the non-comoving phase we are about to describe is very heuristic and mainly used for illustration.

We assume that the antibrane loses most of its kinetic energy only when it moves in the non-comoving region around the IR tip which has the effective warp factor $h_{e}$ due to the antibrane back-reaction. Then it will bounce back (in terms of the radial coordinate $r$ ), and, after a non-comoving phase, enters back to the comoving phase and reaches a maximum point $h=h_{0}$. We connect the different phases smoothly by a straight effective geometry as shown in Fig. 2. Since $h_{e} \ll h_{0}$, most part of this effective geometry is close to the background geometry, except for a small region around the IR end, which effectively describes the non-comoving phase. After the antibrane reaches $h_{0}$, it falls back again due to the background attraction and oscillates in this fashion for many rounds. The values of $h_{e}$ and $h_{0}$ are decreasing due to the loss of energy. We assume that for each round the fraction of the energy loss is small enough so we can approximate the evolution as a smooth function of time.

Using (3.1) and (3.2) in our effective geometry, we see that the time scale of each round is

$$
\Delta t \approx R h_{e}^{-1}
$$

which is dominated by the speed limit behavior (3.2) at small $r$. 


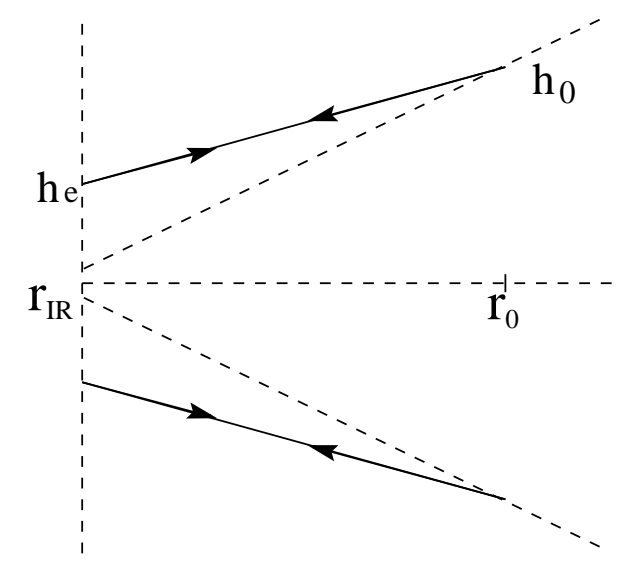

FIG. 2. The solid lines are the effective geometry taking into account of the antibrane back-reaction. $r_{I R}$ is the IR cut-off, $r_{0}$ is the largest $r$-value the antibrane can go. $h_{e}$ and $h_{0}$ are the warp factors of this effective geometry at $r_{I R}$ and $r_{0}$, respectively. Both $h_{e}$ and $h_{0}$ are decreasing while the antibrane oscillates.

On the other hand, the effective warp factor $h_{e}$ is determined by the total antibrane self-energy density around $r_{I R}$. It is convenient to denote this energy density as $\mathcal{F}$ in unit of the static antibrane tension $\left(\sim h_{e}^{4} T_{3}\right)$. Since $\mathcal{F}$ reaches a saturation after the antibrane travels several e-folds, $\mathcal{F}$ cannot decrease significantly before $h_{0}$ reaches several e-folds above the IR tip warp factor $h_{I R}$ of the original background. So for most range of $h_{e}, \mathcal{F}$ changes slowly and we approximate

$$
\mathcal{F} \approx \mathcal{F}_{0}+\beta h_{e}^{\alpha}
$$

where $\alpha, \beta$ are unknown parameters, and $\mathcal{F}_{0}$ is the leading constant. If we further assume that the fraction of the energy loss for each round is the same, ${ }^{3}$ from (5.1) and (5.2) we have

$$
d h_{e} \propto-h_{e}^{-\alpha+2} d t / R
$$

Depending on the value of $\alpha, h_{e}$ decays exponentially or in a power-law.

Since the proper three-volume of the antibrane remains approximately unchanged at the IR tip $r=r_{I R}$, a fixed length scale measured in the world-volume coordinates $\xi^{i}$ appears to be expanding in the Poincare coordinate $x^{i}$ as $h_{e}$ decreases. This is achieved in a purely geometric way. For example, we label a fixed length on the world-volume in the 1-direction by the world-volume coordinate $\Delta \xi^{1}$. For the non-comoving case, the proper length $h_{e} \Delta x^{1}$ (for

\footnotetext{
${ }^{3}$ If the energy loss has some dependence on $h_{e}$, it then changes the value of $\alpha$ in (5.3).
} 
constant $t$ slice) is approximately fixed during the evolution. We can choose the convention to have the relation $\Delta \xi^{1}=h_{e} \Delta x^{1}$. Therefore for the Poincare observer, the metric can be written as $d s^{2}=-d t^{2}+\left(d x^{i}\right)^{2}=-d t^{2}+h_{e}^{-2}\left(d \xi^{i}\right)^{2}$. So the effective scale factor $s(t)$ for the Poincare observer is proportional to $h_{e}(t)^{-1}$, which, for world-volume fields, corresponds to a universe expanding exponentially or in a power-law. (We note that this is not inflation since the background metric (2.1) is not inflating.) This is to be compared with the comoving case where $\Delta x^{1}=\Delta \xi^{1}$ as shown in Sec. II, in which case, $\Delta x^{1}$ is fixed instead of $h_{e} \Delta x^{1}$.

\section{DISCUSSION AND OPEN QUESTIONS}

In this paper we have discussed a novel possibility to generate the brane world-volume homogeneity and flatness. A more detailed understanding of the intermediate evolution is necessary to see how much it can account for the initial conditions of the big bang. For example, density perturbation is crucial to possibly connect this mechanism to observations. Unfortunately we can only make some preliminary remarks here. In inflation, density fluctuations are seeded when the scalar field quantum fluctuations exit the horizon and become classical objects. Higher frequency modes $k$ exit later and are suppressed, due to the expansion, by a factor of $1 / k$ relative to the vacuum spectrum of the flat space. In the rescaling mechanism discussed in this paper, horizon exit can also happen if the rescaling factor $s(t)$ expands fast enough. If this is the case, the horizon exit of the density perturbation and the reheating are happening at the same time - the background warp factor $h_{e}$ restores to $h_{I R}$ at the same time when the anti-D3-branes are losing their kinetic energy to string creation. There is also a suppression for the higher frequency modes - they exit the horizon later and therefore correspond to lower kinetic energy. But at the present stage, it is unclear how much this suppression is in terms of the density perturbation.

It is also possible that the rescaling may be combined with the inflation. Recent studies indicate that, under certain circumstances, brane inflation involving D3-anti-D3-branes $[7,8]$ can happen in a warped space $[9,10]$. In these models, the anti-D3-branes have already settled down in the IR cut-off of the AdS space, while the D3-branes are attracted toward the IR end from the UV entrance. When the D3-branes are in their non-relativistic phase, inflation can happen if the slow roll conditions can be managed, assuming that the endproduct of the brane-anti-brane annihilation leave some anti-D3-branes with a very small cosmological constant [5]. Inflation may also happen with anti-D3-branes alone at the IR end [18]. Both the D3 and anti-D3-branes may have their non-comoving phase. The rescaling mechanism takes place before the inflation for anti-D3-branes, and after the inflation for D3branes if they start fast-rolling. This may reduce the required large inflationary e-foldings. 
Matter (fundamental open strings) can be created on the anti-D3-branes through various processes. With the anti-D3-branes alone, we have seen that they gain kinetic energy from the comoving region. When they interact with the IR end of the background (or collide with themselves if there are already some antibranes in the IR end), presumably part of energy will be used to create the open strings on the anti-D3-branes. After they settle down, the open string tension is red-shifted by the corresponding warp factor, namely $h_{I R}^{2} \alpha^{\prime}$, and the total energy density of the open strings created cannot exceed the kinetic energy density of the accelerated anti-D3-brane, which is of order $N h_{I R}^{4} T_{3}$ according to Sec. II \& III. If we consider the abovementioned D3-anti-D3-brane system, D3 branes may gain similar kinetic energy and transfer part of it to the anti-D3-branes. In addition, when D3 and anti-D3branes annihilate, strings can also be created through the rolling tachyon [19]. The tree level diagram dumps energy into the bulk in terms of the massive closed strings [20], while loop diagrams connecting the rolling tachyon and anti-D3-branes can create massive open strings on the anti-D3-branes [21]. The total energy density of the open strings cannot exceed the red-shifted (anti)brane tension $n h_{I R}^{4} T_{3}$ where $n$ includes all the (anti)branes annihilated. Overall we end up with open strings with red-shifted tension and total energy density at most of order $N h_{I R}^{4} T_{3}$. This should be responsible for the subsequent cosmological big bang.

\section{ACKNOWLEDGMENTS}

This work was supported in part by the Department of Energy under Grant No. DEFG02-97ER-41029. 


\section{REFERENCES}

[1] L. Randall and R. Sundrum, Phys. Rev. Lett. 83, 3370 (1999) [arXiv:hep-ph/9905221].

[2] H. Verlinde, Nucl. Phys. B 580, 264 (2000) [arXiv:hep-th/9906182].

[3] I. R. Klebanov and M. J. Strassler, JHEP 0008, 052 (2000) [arXiv:hep-th/0007191].

[4] S. B. Giddings, S. Kachru and J. Polchinski, Phys. Rev. D 66, 106006 (2002) [arXiv:hepth/0105097].

[5] S. Kachru, R. Kallosh, A. Linde and S. P. Trivedi, Phys. Rev. D 68, 046005 (2003) [arXiv:hep-th/0301240].

[6] C. Escoda, M. Gomez-Reino and F. Quevedo, JHEP 0311, 065 (2003) [arXiv:hepth/0307160].

C. P. Burgess, R. Kallosh and F. Quevedo, JHEP 0310, 056 (2003) [arXiv:hepth/0309187].

F. Denef, M. R. Douglas and B. Florea, JHEP 0406, 034 (2004) [arXiv:hep-th/0404257].

[7] G. R. Dvali and S. H. H. Tye, Phys. Lett. B 450, 72 (1999) [arXiv:hep-ph/9812483].

[8] G. R. Dvali, Q. Shafi and S. Solganik, arXiv:hep-th/0105203.

C. P. Burgess, M. Majumdar, D. Nolte, F. Quevedo, G. Rajesh and R. J. Zhang, JHEP 0107, 047 (2001) [arXiv:hep-th/0105204].

[9] S. Kachru, R. Kallosh, A. Linde, J. Maldacena, L. McAllister and S. P. Trivedi, JCAP 0310, 013 (2003) [arXiv:hep-th/0308055].

[10] J. P. Hsu, R. Kallosh and S. Prokushkin, JCAP 0312, 009 (2003) [arXiv:hepth/0311077].

A. Buchel and R. Roiban, Phys. Lett. B 590, 284 (2004) [arXiv:hep-th/0311154].

H. Firouzjahi and S. H. H. Tye, Phys. Lett. B 584, 147 (2004) [arXiv:hep-th/0312020].

C. P. Burgess, J. M. Cline, H. Stoica and F. Quevedo, arXiv:hep-th/0403119.

N. Iizuka and S. P. Trivedi, arXiv:hep-th/0403203.

M. Berg, M. Haack and B. Kors, arXiv:hep-th/0404087.

A. Buchel and A. Ghodsi, arXiv:hep-th/0404151.

[11] A. H. Guth, Phys. Rev. D 23, 347 (1981).

A. D. Linde, Phys. Lett. B 108, 389 (1982).

A. Albrecht and P. J. Steinhardt, Phys. Rev. Lett. 48, 1220 (1982).

[12] M. Gasperini and G. Veneziano, Astropart. Phys. 1, 317 (1993) [arXiv:hep-th/9211021].

[13] J. Khoury, B. A. Ovrut, P. J. Steinhardt and N. Turok, Phys. Rev. D 64, 123522 (2001) [arXiv:hep-th/0103239].

R. Kallosh, L. Kofman and A. D. Linde, Phys. Rev. D 64, 123523 (2001) [arXiv:hepth/0104073]. 
[14] D. J. H. Chung and K. Freese, Phys. Rev. D 62, 063513 (2000) [arXiv:hep-ph/9910235].

[15] E. Silverstein and D. Tong, arXiv:hep-th/0310221.

[16] O. Aharony, S. S. Gubser, J. M. Maldacena, H. Ooguri and Y. Oz, Phys. Rept. 323, 183 (2000) [arXiv:hep-th/9905111].

[17] D. Kabat and G. Lifschytz, JHEP 9905, 005 (1999) [arXiv:hep-th/9902073].

[18] L. Pilo, A. Riotto and A. Zaffaroni, arXiv:hep-th/0401004.

M. R. Garousi, M. Sami and S. Tsujikawa, arXiv:hep-th/0402075.

O. DeWolfe, S. Kachru and H. Verlinde, JHEP 0405, 017 (2004) [arXiv:hepth/0403123].

[19] A. Sen, JHEP 0204, 048 (2002) [arXiv:hep-th/0203211].

A. Sen, JHEP 0207, 065 (2002) [arXiv:hep-th/0203265].

[20] N. Lambert, H. Liu and J. Maldacena, arXiv:hep-th/0303139.

D. Gaiotto, N. Itzhaki and L. Rastelli, Nucl. Phys. B 688, 70 (2004) [arXiv:hepth/0304192].

[21] X. g. Chen, arXiv:hep-th/0311179. 